\title{
Dendritic cells as potential initiators of immune-mediated hypertensive disorders
}

\author{
Akinori Higaki $^{1} \cdot$ Masaki Mogi $^{2}$ \\ Received: 16 November 2021 / Accepted: 19 November 2021 / Published online: 27 December 2021 \\ (C) The Japanese Society of Hypertension 2021
}

Current knowledge suggests the involvement of both innate and adaptive immunity in the pathophysiology of hypertension and hypertension-mediated organ damage (HMOD) [1]. Antigen-presenting cells (APCs), such as dendritic cells (DCs), play a pivotal role in the initiation of adaptive immunity, which may contribute to vascular and kidney injury in hypertension through $\mathrm{T}$ cell activation [2]. DCs are mainly derived from hematopoietic bone marrow progenitor cells and exist in an immature state in blood. After exposure to pathogens, DCs are activated to the mature form and migrate to lymph nodes with the capability to induce an adaptive immune response. Peripheral DCs are divided into two subsets, namely, myeloid DCs (mDCs) and plasmacytoid DCs (pDCs). The former are characterized by CD11c expression and have a high capacity to produce proinflammatory cytokines (e.g., interleukin (IL)-6, IL-12 and IL-23), while the latter express CD123 and preferentially secrete type I interferon (IFN) to protect against viral infection. Both subsets of DCs play roles not only in infectious diseases but also in the development of autoimmune diseases, such as rheumatoid arthritis and systemic lupus erythematosus. However, the distribution of DC subsets may vary from disease to disease. An $\mathrm{mDC} / \mathrm{pDC}$ imbalance was reported in coronary heart disease patients by Shi et al. in 2007 [3], but its significance has not been rigorously evaluated in the context of hypertension.

In the present study, Kubiszewska and colleagues compared the profile of DC between hypertensive and normotensive adolescents. Using flow cytometric analysis, they found that hypertensive subjects have a significantly higher percentage

Masaki Mogi

mmogi@m.ehime-u.ac.jp

1 Department of Cardiology, Ehime Prefectural Central Hospital, Matsuyama, Japan

2 Department of Department of Pharmacology, Ehime University Graduate School of Medicine, Tohon, Japan of mDCs and a lower percentage of pDCs than normotensive subjects [4]. The strength of their study is that they showed an association between various clinical data, including hemodynamic indices, and immune cell phenotypes. As data from younger patients are limited in this field, they provide important insights for our discussion of the mechanisms underlying the early development of hypertension. Previously, Hevia et al. demonstrated that genetic ablation of CD11 $\mathrm{c}^{\text {high }}$ APCs (i.e., mDCs) reduced blood pressure in mice treated with angiotensin (Ang) II plus high salt. Deletion of mDCs also prevented the decrease in IL-10 and FoxP3 mRNA expression in response to AngII plus salt treatment [5]. Therefore, the present result that CD11c-positive DCs are predominant in hypertensive children provides a clue for a therapeutic target to prevent HMOD. Since eliminating all dendritic cells would be harmful to the entire body, we need to find more selective therapeutic target molecules.

In the last decade, the role of A20, a zinc finger protein and ubiquitin-editing enzyme, has also been highlighted in the mechanism of DC-mediated autoimmunity. Kool et al. showed that A20-deficient mice with an inflammatory phenotype had increased conventional (myeloid) DCs, whereas the number of pDCs was decreased [6]. More recently, $\mathrm{Lu}$ and colleagues reported that specific deletion of A20 in CD11c-positive myeloid cells enhanced the accumulation of effector memory $\mathrm{T}$ cells in the kidney, which could promote blood pressure elevation [7]. In this study, the effect of A20 deletion was more marked in CD8positive $\mathrm{T}$ cells than in CD4-positive $\mathrm{T}$ cells. In any case, A20 serves as an antigen presentation attenuator, and downregulation of A20 may serve as the underlying factor in DC-associated hypertension.

When considering the relation between innate immunity and hypertension, sodium intake is one of the most important factors [8]. Recently, Barbaro found that extracellular sodium enters DCs through amiloride-sensitive channels, leading to calcium overload in DCs. Activated DCs then produce superoxides, which induce the formation 
Fig. 1 Imbalance of dendritic cell subsets and its contribution to $\mathrm{T}$ cell-mediated hypertension. DC dendritic cell, pDC plasmocytoid $\mathrm{DC}, \mathrm{mDC}$ myeloid $\mathrm{DC}$, Treg regulatory $\mathrm{T}$ cell, Th17 interleukin-17producing helper $\mathrm{T}$ cell, $\mathrm{ARB}$ angiotensin II type 1 receptor blocker, ERK extracellular signal-regulated kinase, NF-kB nuclear factor-kappa B, IL-23 interleukin-23, HMOD hypertension-mediated organ damage, PWV pulse wave velocity

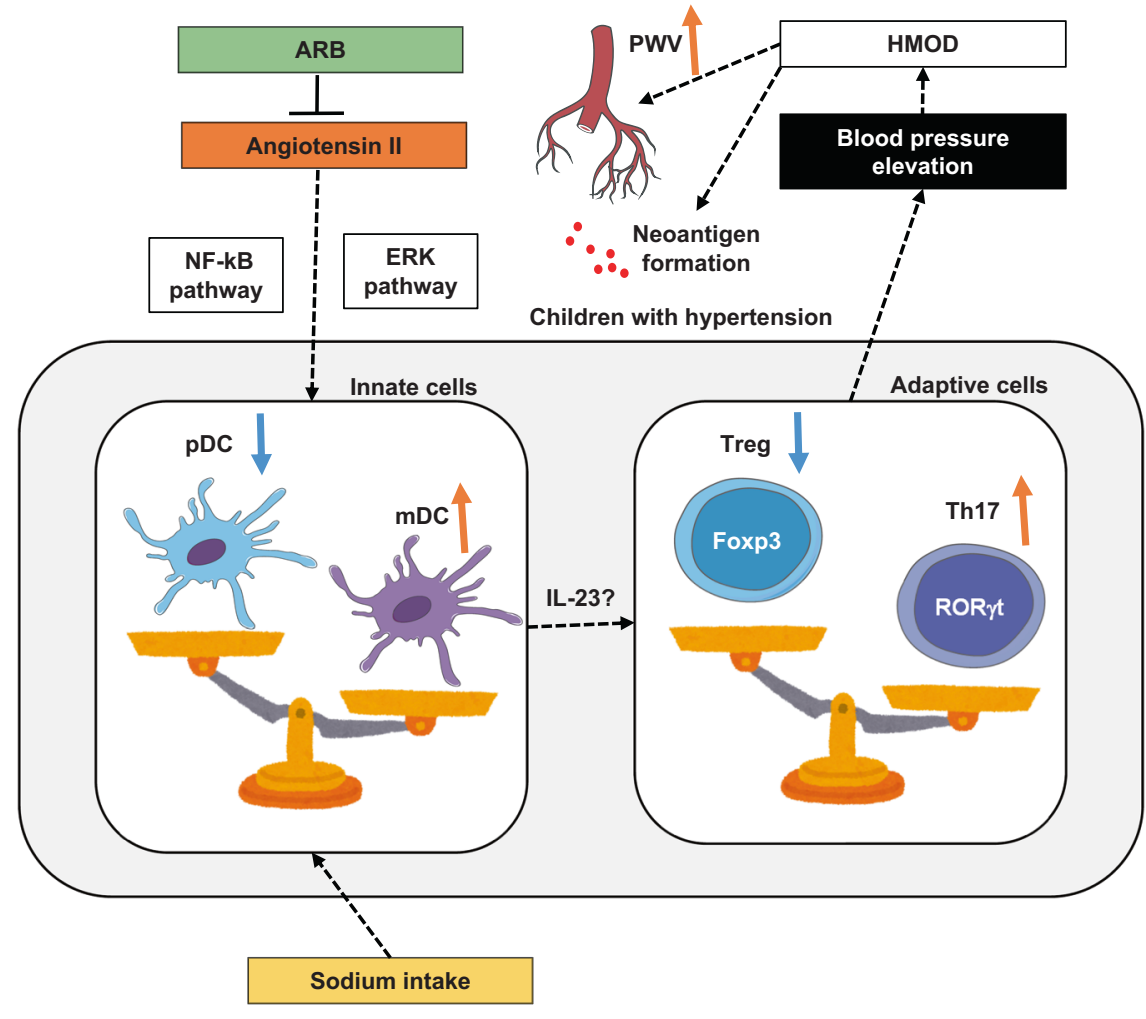

of neoantigens (e.g., isolevuglandins) that are presented to T cells [9]. In the present study, the authors showed that the hypertensive population has a higher percentage of IL-17producing helper $\mathrm{T}$ cells (Th17) than their normotensive counterparts. It is conceivable that hypertensive subjects are more likely to produce neoantigens by activated DCs, which in turn promote $\mathrm{T}$ cell immunity, leading to a vicious cycle of inflammatory response and HMOD. T cell activation can occur in either a TCR-dependent or cytokinemediated manner.

Pulse wave velocity (PWV) is a marker of arterial stiffening and HMOD [10] and is correlated with the level of isoketal adducts in human aortic tissue [11]. The present study demonstrated that PWV was significantly higher in the hypertensive population and was positively correlated with increased expression of DC maturation/activation markers. Carotid artery intima-media thickness was also significantly higher in the primary hypertension group than in normotensive subjects. These results are in line with the recent report by del Mar Vila et al. showing that individuals with autoimmune disease have an increased risk of subclinical carotid atherosclerosis and stiffness [12]. In the present study, even the white-coat hypertension group showed significantly higher PWV than the normotensive group. In addition, certain DC characteristics in white-coat hypertension children resemble those of primary hypertension subjects, such as an increase in CD83-positive DCs and loss of CD86-positive pDCs. An interesting point here might be that the white-coat hypertension subjects had the highest body mass index compared to the other groups. The authors raised this issue as a main limitation of the present study. However, this finding seems to reflect the characteristics of young hypertensive patients in Poland and is rather suggestive in understanding the pathogenesis. Obesity is correlated with sympathetic nerve activity. It has been reported that sympathetic nerve hyperactivity precedes blood pressure elevation and usually exists in white-coat hypertension [13]. Therefore, DC induction may occur at an early stage of hypertensive disease and contribute to disease progression.

However, as stated in the Discussion, it is impossible to determine the causal relationship between altered distribution of DC and a hypertensive state from the presented results. Whether the skew in DC distribution can drive immune-mediated hypertension will only be clarified through interventional studies. As there is evidence that AngII directly and indirectly activates DCs via the ERK pathway [14] and NF kappa B pathway [15], the effect of AngII receptor blockers on the $\mathrm{mDC} / \mathrm{pDC}$ ratio is of interest (Fig. 1). In addition, the relationship between gut microbiota and DC distribution in hypertensive patients is also an interesting research topic. Finally, since the sympathetic nervous system is associated with all aspects of hypertension etiology, including gut dysbiosis, future studies are expected to determine how renal denervation alters the circulating DC phenotype in human samples. 


\section{Compliance with ethical standard}

Conflict of interest The authors declare no competing interests.

Publisher's note Springer Nature remains neutral with regard to jurisdictional claims in published maps and institutional affiliations.

\section{References}

1. Solak Y, Afsar B, Vaziri ND, Aslan G, Yalcin CE, Covic A, et al. Hypertension as an autoimmune and inflammatory disease. Hypertens Res. 2016;39:567-73.

2. Caillon A, Paradis P, Schiffrin EL. Role of immune cells in hypertension. Br J Pharm. 2019;176:1818-28.

3. Shi H, Ge J, Fang W, Yao K, Sun A, Huang R, et al. Peripheralblood dendritic cells in men with coronary heart disease. Am J Cardiol. 2007;100:593-7.

4. Kubiszewska I, Gackowska L, Obrycki Ł, Wierzbicka A, HelminBasa A, Kułaga Z, et al. Distribution and maturation state of peripheral blood dendritic cells in children with primary hypertension. Hypertens Res. 2021. https://doi.org/10.1038/s41440-021-00809-9.

5. Hevia D, Araos P, Prado C, Fuentes Luppichini E, Rojas M, Alzamora R, et al. Myeloid CD11c(+) antigen-presenting cells ablation prevents hypertension in response to angiotensin II plus high-salt diet. Hypertension. 2018;71:709-18.

6. Kool M, van Loo G, Waelput W, De Prijck S, Muskens F, Sze M, et al. The ubiquitin-editing protein A20 prevents dendritic cell activation, recognition of apoptotic cells, and systemic autoimmunity. Immunity. 2011;35:82-96.

7. Lu X, Rudemiller NP, Wen Y, Ren J, Hammer GE, Griffiths R, et al. A20 in myeloid cells protects against hypertension by inhibiting dendritic cell-mediated T-cell activation. Circ Res. 2019;125:1055-66.

8. Targonski R, Sadowski J, Price S, Targonski R. Sodium-induced inflammation-an invisible player in resistant hypertension. Hypertens Res. 2020;43:629-33.

9. Barbaro NR, Foss JD, Kryshtal DO, Tsyba N, Kumaresan S, Xiao $\mathrm{L}$, et al. Dendritic cell amiloride-sensitive channels mediate sodium-induced inflammation and hypertension. Cell Rep. 2017;21:1009-20.

10. Sougawa Y, Miyai N, Utsumi M, Miyashita K, Takeda S, Arita M. Brachial-ankle pulse wave velocity in healthy Japanese adolescents: reference values for the assessment of arterial stiffness and cardiovascular risk profiles. Hypertens Res. 2020;43:331-41.

11. Wu J, Saleh MA, Kirabo A, Itani HA, Montaniel KR, Xiao L, et al. Immune activation caused by vascular oxidation promotes fibrosis and hypertension. J Clin Invest. 2016; 126:50-67.

12. Vila MDM, Remeseiro B, Igual L, Elosua R, Ramos R, Valdivielso JM, et al. Do individuals with autoimmune disease have increased risk of subclinical carotid atherosclerosis and stiffness? Hypertens Res. 2021;44:978-87.

13. Smith PA, Graham LN, Mackintosh AF, Stoker JB, Mary DA. Sympathetic neural mechanisms in white-coat hypertension. J Am Coll Cardiol. 2002;40:126-32.

14. Yang L, Du C, Chen T, Li S, Nie W, Zhu W, et al. Distinct MAPK pathways are involved in IL-23 production in dendritic cells cocultured with NK cells in the absence or presence of angiotensin II. Mol Immunol. 2012;51:51-6.

15. Zhao H, Li M, Wang L, Su Y, Fang H, Lin J, et al. Angiotensin II induces TSLP via an AT1 receptor/NF-KappaB pathway, promoting Th17 differentiation. Cell Physiol Biochem. 2012;30:1383-97. 